\title{
Influência do tipo de solvente na síntese de mulita pelo método Pechini
}

\section{(Influence of the solvent type on the synthesis of mullite by the Pechini method)}

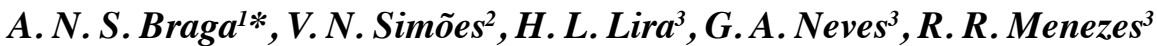 \\ ${ }^{I}$ Universidade Federal do Piauí, Av. Universitária, s/n, Teresina, PI, Brasil \\ ${ }^{2}$ Universidade Federal do Rio Grande do Norte, Natal, RN, Brasil \\ ${ }^{3}$ Universidade Federal de Campina Grande, Campina Grande, PB, Brasil
}

\begin{abstract}
Resumo
Mulita é um aluminossilicato com grande importância na tecnologia cerâmica. Sua síntese através de métodos químicos tem sido extensivamente estudada, no entanto, não se observam estudos da sua obtenção pelo método Pechini. Assim, este estudo teve por objetivo a síntese de mulita por meio deste método. Foi avaliada a influência de diversos solventes na obtenção da mulita pela rota Pechini. As características dos pós foram avaliadas por difração de raios X, análise térmica (TG/DTG), adsorção de nitrogênio (BET), microscopia eletrônica de varredura e de transmissão. Os resultados obtidos comprovaram a eficiência do método Pechini, bem como a influência dos solventes na obtenção da fase, sendo a mulita obtida como fase majoritária e com maior cristalinidade quando do uso do álcool etílico como solvente.
\end{abstract}

Palavras-chave: síntese, método Pechini, mulita.

\begin{abstract}
Mullite is an aluminosilicate with great importance in ceramic technology. Its synthesis through chemical methods has been extensively studied, however, no studies of its obtention by the Pechini method are observed. Thus, this work aimed to synthesize mullite using this method. The influence of several solvents in obtaining the mullite by the Pechini route was evaluated. The characteristics of the powders were evaluated by X-ray diffraction, thermal analysis (TG/DTG), nitrogen adsorption (BET), scanning and transmission electron microscopy. The results obtained proved the efficiency of the Pechini method, as well as the influence of the solvents in obtaining the phase, being the mullite obtained as the majority phase and with greater crystallinity when using ethyl alcohol as a solvent.
\end{abstract}

Keywords: synthesis, Pechini method, mullite.

\section{INTRODUÇÃO}

Há um interesse considerável nas últimas décadas no estudo e aplicação da mulita, devido às suas destacadas propriedades, dentre elas: baixa expansão térmica $\left(4.10^{-6} \mathrm{~K}^{-1}\right.$ entre 20 a $200^{\circ} \mathrm{C}$ ), alta estabilidade térmica, baixa densidade $\left(3,17 \mathrm{~g} / \mathrm{cm}^{3}\right)$, baixa condutividade térmica $\left(2,0 \mathrm{~W} \cdot \mathrm{m}^{-1} \cdot \mathrm{K}^{-1}\right)$, boa resistência mecânica e resistência à fluência, e boa estabilidade em ambientes químicos severos [1-4]. Nesse sentido, estudos na última década [5-11] vêm abordando a síntese de mulita, avaliando diferentes metodologias de síntese, o uso de diferentes precursores e proporções de $\mathrm{Si}$ e Al na síntese e a influência de dopantes, sendo o método sol-gel a rota de síntese química mais utilizada na síntese da mulita. A versatilidade deste método é uma de suas maiores vantagens, podendo obter óxidos cerâmicos na forma de pós nanométricos, submicrométricos e micrométricos [12-15].

*aluskasimoes@hotmail.com

(D) https://orcid.org/0000-0002-7764-6147
A técnica sol-gel pode ser executada através de diferentes soluções, sejam elas coloidais ou poliméricas, ou através da metodologia Pechini [16-18], uma variação da rota sol-gel.

Este método foi proposto por Pechini em 1967 para formar uma fina camada dielétrica de alta pureza em um substrato metálico para fabricação de capacitores [19]. Desde então, o método Pechini tem sido utilizado para a síntese de vários pós cerâmicos e filmes para diversas aplicações. Neste método, precursores metálicos são dissolvidos numa mistura de um álcool poli-hidróxi e um ácido carboxílico multifuncional. Normalmente, o ácido cítrico (CA) e o etilenoglicol (EG) são utilizados como o ácido carboxílico multifuncional e o álcool poli-hidróxi, respectivamente. Um complexo metal-citrato é formado pela quelação de íons metálicos por CA e uma reação de poliesterificação entre o complexo metal-citrato e EG leva à rede tridimensional de polímeros com complexos metálicos incorporados e misturados na escala atômica [20, 21]. O método Pechini, em relação a outras técnicas, apresenta vantagens, tais como homogeneidade química dos multicomponentes em escala 
atômica, temperaturas de calcinação relativamente baixas, controle direto e preciso da estequiometria de sistemas complexos, pós cerâmicos com partículas muito finas, simplicidade de processamento e alta reprodutibilidade [20, 22, 23].

Por outro lado, apesar das inúmeras pesquisas envolvendo a tecnologia sol-gel, têm-se uma certa escassez na literatura de estudos aplicando o método Pechini para obtenção de mulita. Na tecnologia sol-gel a escolha da rota, das variáveis de síntese e do tipo do solvente afeta diretamente as propriedades do produto final obtido, interferindo significativamente na homogeneidade química do sistema e nas características microestruturais do material obtido [24-30]. Assim, os pós obtidos pelo método Pechini apresentam características particulares que os diferenciam de materiais sintetizados por outras variantes do método solgel. Deste modo, frente à carência de estudos na literatura envolvendo a produção de mulita pelo método Pechini, às particularidades do método e à importância deste material, este estudo teve por objetivo a síntese da mulita pelo método Pechini, analisando a influência do tipo de solvente na formação das fases.

\section{MATERIAIS E MÉTODOS}

A mulita foi preparada pelo método Pechini utilizando como precursores nitrato de alumínio nano-hidratado (NAN, Neon, 98\%) e tetraetilortossilicato (TEOS, Fluka, 99\%). Os precursores foram utilizados de modo a se obter uma proporção de Al:Si de 3:1. Foram utilizados como solventes: mistura 50:50 de água e álcool etílico (Santa Cruz, PA); água com $\mathrm{pH}$ ácido ( $\mathrm{pH} \sim 2$, corrigido com $\mathrm{HCl}$, Fmaia); água com $\mathrm{pH}$ básico ( $\mathrm{pH} \sim 12$, corrigido com $\mathrm{NH}_{4} \mathrm{OH}$, Vetec), denominada Ag-Ba; álcool etílico (Santa Cruz, PA); e álcool isopropílico (Cinética, PA). Foi utilizado ácido cítrico mono-hidratado (AC, Neon, 99\%), e etileno glicol (EG, Synth, 99\%). Os solventes foram aquecidos a $70{ }^{\circ} \mathrm{C}$ e o CA foi adicionado e dissolvido nos solventes. Posteriormente, os precursores NAN e TEOS foram adicionados. Foi utilizada a razão $\mathrm{CA} /$ cátion metálico de 1:1. O sistema foi agitado por $8 \mathrm{~h}$ e em seguida adicionado o etileno glicol, sob agitação contínua, aumentando-se a temperatura da placa até $120^{\circ} \mathrm{C}$. Em seguida ocorreram as reações de esterificação e poliesterificação, formando o gel. O gel foi então calcinado a $450{ }^{\circ} \mathrm{C} / 2 \mathrm{~h}$, com taxa de aquecimento de $5{ }^{\circ} \mathrm{C} / \mathrm{min}$, para eliminação da matéria orgânica. Em seguida o pó obtido foi desaglomerado em um almofariz, passado em peneira ABNT n $200(0,074 \mathrm{~mm})$, e queimado a $1200{ }^{\circ} \mathrm{C}$ durante 2 $\mathrm{h}$, com taxa de aquecimento de $5^{\circ} \mathrm{C} / \mathrm{min}$, para obtenção da fase desejada. As amostras foram denominadas de Ag-Etil, Ag-Ac, Ag-Ba, Etil, Iso, quando se utilizou como solvente água+álcool etílico, água acidificada, água basificada, álcool etílico e álcool isopropílico, respectivamente.

Os materiais foram analisados por difração de raios X (Shimadzu, XRD-6000, CuKa) para identificação das fases presentes e grau de cristalização. Na identificação das amostras foram utilizadas fichas do Joint Committee on Powder Diffraction Standards (JCPDS), banco de dados PCPDFWIN: JCPDS 15-0776 para mulita, JCPDS 10-0173 para $\alpha$-alumina e JCPDS 37-1483 para os picos de espinélio. A cristalinidade foi determinada a partir da razão entre a área integrada do pico referente à fase cristalina e a área referente à fração amorfa (Shimadzu, software Search Match). Os materiais foram caracterizados também por: análise termogravimétrica (TG, Shimadzu, TA-60), em atmosfera de ar e taxa de aquecimento de $10{ }^{\circ} \mathrm{C} / \mathrm{min}$; determinação de área superficial por isotermas de adsorção/dessorção de $\mathrm{N}_{2}$ (Micromeritics, ASAP 2020); microscopia eletrônica de varredura (MEV, Philips, XL-30 FEG); e microscopia eletrônica de transmissão (MET, Philips, CM-120).

\section{RESULTADOS E DISCUSSÃO}

A Fig. 1 ilustra os difratogramas de raios $\mathrm{X}$ das amostras sintetizadas pelo método Pechini, utilizando como solventes água+álcool etílico, água acidificada, água basificada, álcool etílico e álcool isopropílico, denominadas de amostras AgEtil, Ag-Ac, Ag-Ba, Etil e Iso, respectivamente. Os padrões de difração das amostras evidenciaram que a utilização da água como solvente, independentemente do $\mathrm{pH}$ utilizado, não favoreceu a formação da fase mulita. A amostra Ag-Etil apresentou a formação de discretos picos referentes ao início da cristalização da mulita juntamente com a formação de $\alpha$-alumina, picos de espinélio e bandas amorfas, com uma cristalinidade de 41,1\%. Já as amostras Ag-Ac e Ag-Ba, sintetizadas em meio ácido e básico, apresentaram um perfil semelhante, ou seja, a formação de picos característicos da fase alumina e formação de $\mathrm{SiO}_{2}$, bandas referentes ao material amorfo e reflexões associadas à formação de espinélio, com cristalinidade de $42,4 \%$ e $51,0 \%$ para as amostras $\mathrm{Ag}-\mathrm{Ac}$ e $\mathrm{Ag}-\mathrm{Ba}$, respectivamente. Observou-se uma dificuldade na cristalização da fase mulita para todas as amostras que utilizaram água como solvente. A maioria das pesquisas envolvendo a síntese de mulita utilizando TEOS como precursor utiliza como solvente misturas álcool/água ou apenas álcool para a síntese [10,31-33], pois a presença de água provoca a rápida hidrólise do TEOS favorecendo a formação de oligômeros de sílica, que segregam, dificultando sua interação com o alumínio, dificultando assim a cristalização da mulita, formando grandes bandas amorfas. A literatura [34-36] relata que se o teor de água exceder um determinado valor, a homogeneidade da solução é perdida, devido à precipitação. Quanto maior a quantidade de água no sistema reacional da síntese, menor a sua homogeneidade e maior a temperatura de cristalização da mulita. Para menores teores de água, as espécies moleculares parcialmente hidrolisadas são solúveis. As reações de condensação entre as espécies parcialmente hidrolisadas levam à produção de um gel homogêneo.

Apesar disto, vários estudos [28, 37, 38] utilizam soluções aquosas para síntese de mulita pelo método sol-gel, porém utilizando agentes quelantes, que retardam a hidrólise do alcóxido de modo a melhorar a homogeneidade química do gel. Desta forma, a síntese de mulita pelo método Pechini 


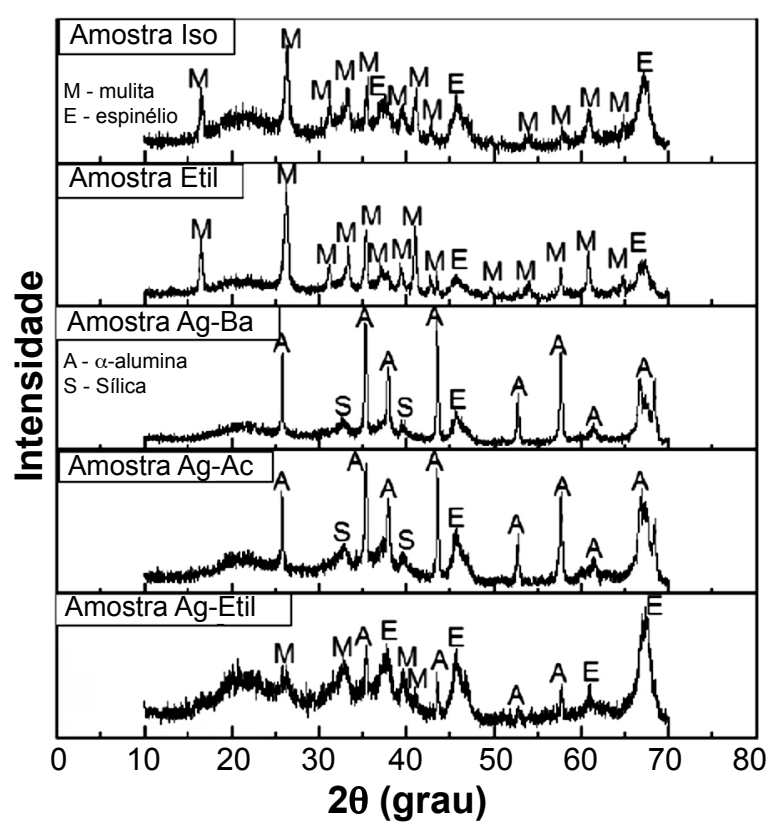

Figura 1: Difratogramas de raios $\mathrm{X}$ das amostras Ag-Etil, Ag-Ac, Ag-Ba, Etil e Iso.

[Figure 1: X-ray diffraction patterns of samples Ag-Ethyl, Ag-Ac, Ag-Ba, Ethyl and Iso.]

deveria ter apresentado boa homogeneidade, devido à presença do AC que age como quelante dos íons metálicos. Porém, apesar da ação quelante do AC, não foi possível uma mistura homogênea da solução. Sabe-se que os íons metálicos em solução interagem com o AC em maior ou menor grau, onde este grau de interação é medido pela relação entre os íons quelados e os íons livres. O alumínio apresenta alta interação com o AC, enquanto o silício apresenta baixa interação. Desta forma, o que possivelmente ocorreu nesta reação dificultando a formação da mulita foi que o íon alumínio formou o quelante com o AC, enquanto o TEOS foi hidrolisado nos sistemas contendo maior quantidade de água e permaneceu na solução (na forma de oligômeros de sílica); com isso, os íons de silício e alumínio foram separados durante a síntese quando da adição do EG e formação da rede polimérica tridimensional, características do método Pechini, ao invés de serem unidos (em um nível atômico). Diante disto, nos sistemas com muita água, os íons alumínio formaram o quelante com o AC, porém o alumínio não foi totalmente quelado, possuindo sítios reativos que reagiram com pequenas cadeias de TEOS hidrolisado e com grandes oligômeros de sílica, formando no material final espinélio e fase amorfa de sílica, cristalizando alguns núcleos de mulita e alumina, oriunda do alumínio quelado que estava segregado nos oligômeros de sílica. Nos sistemas com pequena quantidade de água que utilizaram como solvente álcool etílico (Etil) e álcool isopropílico (Iso), observouse a formação de mulita em grande quantidade, em virtude de não ter ocorrido uma hidrólise acentuada do TEOS e a segregação dos íons durante o processo de formação do sol e de gelificação. O sistema Etil, com uma menor quantidade de água, apresentou um desenvolvimento maior de mulita que o Iso, o que destacou que mesmo pequenas quantidades de água, quando se usa o TEOS no método Pechini, podem provocar alterações na evolução microestrutural do sistema.

As pesquisas [39-42] envolvendo o método Pechini utilizam água como solvente, no entanto, no presente estudo verificou-se que a utilização de álcool etílico foi mais eficiente, ocorrendo a cristalização da mulita, cuja amostra Etil apresentou uma cristalinidade de $51,6 \%$, com formação de discretos picos de espinélio. Nesta reação, quando a quantidade de água foi diminuída, os íons de alumínio quelados pelo $\mathrm{AC}$, que possuíam sítios reativos, reagiram com a sílica não mais segregada, formando a mulita. Assim, quando o excesso de água foi eliminado e o solvente usado foi apenas o álcool etílico, as reações químicas foram facilitadas, dificultando a segregação de fases, melhorando a homogeneidade do sistema e possibilitando a formação da mulita. Analisando o difratograma da amostra Iso, percebeu-se que esta possuiu um padrão bastante amorfo, com formação de picos que foram referentes à fase mulita e ao espinélio; a cristalinidade da amostra foi de $37,4 \%$. Comparando esta síntese que usou álcool isopropílico (amostra Iso) com a síntese que usou álcool etílico (amostra Etil), observaram-se padrões de difração semelhantes, onde ambas formaram as mesmas fases (mulita e espinélio), porém a amostra sintetizada com álcool etílico apresentouse mais cristalina.

Os resultados obtidos neste estudo comprovaram que a água não é adequada para ser usada como solvente na síntese de mulita pelo método Pechini e que ela deve ser usada em uma quantidade mínima, apenas para a fase da hidrólise, em concordância com dados da literatura [26, 43], que indicam que a água do próprio NAN (precursor do $\mathrm{Al}$ ) é suficiente para a hidrólise do TEOS. De acordo com os difratogramas de raios $\mathrm{X}$ de todas as amostras obtidas pelo método Pechini, apenas a amostra Etil, que usou álcool etílico como solvente, obteve mulita como fase majoritária, apresentando a maior cristalinidade. Desta forma, apenas nesta amostra deu-se prosseguimento às demais caracterizações.

A Fig. 2 apresenta as curvas de TG/DTG da amostra Etil em função da temperatura. Pôde-se observar que a curva referente à $\mathrm{TG}$ apresentou três eventos de perda de massa. $\mathrm{O}$ primeiro ocorreu aproximadamente na faixa de 69 a $163{ }^{\circ} \mathrm{C}$ e o segundo na faixa de 163 a $315^{\circ} \mathrm{C}$, ambos corresponderam à perda de água residual do gel e do álcool ainda presente no material. O terceiro, que ocorreu na faixa de 315 a $522{ }^{\circ} \mathrm{C}$, foi referente à decomposição dos componentes do gel e da queima do material orgânico liberando óxidos de carbono e nitrogênio na forma de gás (a matéria orgânica presente no material foi relacionada ao $\mathrm{AC}$ e ao EG adicionados ao sistema, além do material advindo do TEOS). A partir de $522{ }^{\circ} \mathrm{C}$ a perda de massa permaneceu constante e o material teve uma perda de massa total de aproximadamente $92 \%$.

O resultado da caracterização textual da amostra Etil está representado pela isoterma de adsorção/dessorção de nitrogênio na Fig. 3, que exibiu um ciclo de histerese 


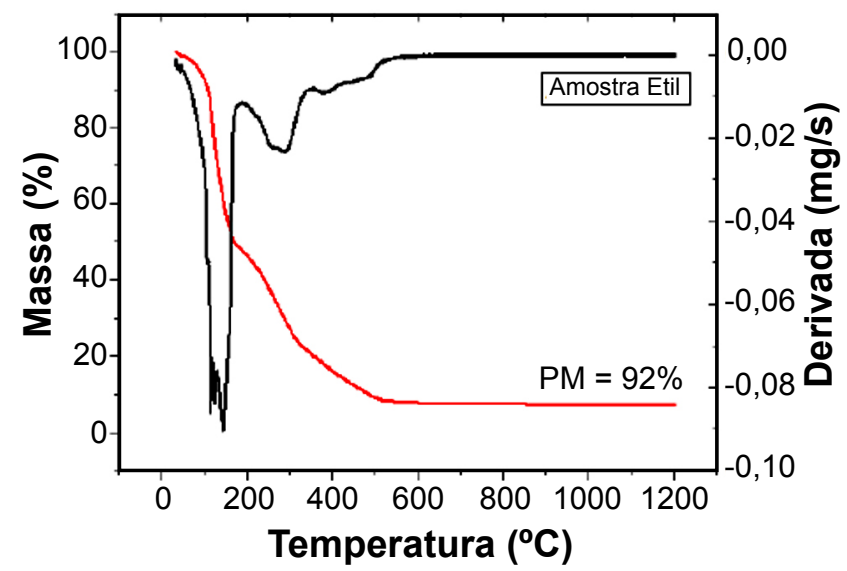

Figura 2: Curvas de TG/DTG da amostra Etil sintetizada usando álcool etílico como solvente.

[Figure 2: TG/DTG curves of the sample Ethyl synthesized using ethyl alcohol as the solvent.]

relacionado à condensação capilar nos mesoporos [44-46] e teve um limite de adsorção em elevada relação P/Po. Isso indicou, apesar da falta de perfeita associação, de se tratar de uma isoterma tipo IV, conforme classificação IUPAC [47], com um loop de histerese tipo H3 [44-46], característica de material mesoporoso (diâmetro médio dos poros entre $20 \mathrm{e}$ $500 \AA$ ). O loop de histerese apresentado na isoterma ocorreu entre 0,46 e $0,99 \mathrm{P} / \mathrm{Po}$, o que indicou a presença de poros de dimensões meso, que estão associados em geral a agregados não rígidos de partículas [48-50]. A amostra apresentou um elevado valor de área superficial específica $\left(33,44 \mathrm{~m}^{2} / \mathrm{g}\right)$ quando comparado com outros estudos que obtiveram mulita por sol-gel [27, 51]. Provavelmente este maior valor de área superficial específica obtido pelo método Pechini foi resultado da maior quantidade de matéria orgânica usada nesta rota, conduzindo a uma estrutura mais porosa após o processo de decomposição. O valor médio de diâmetro dos poros foi de $209 \AA$ Å, sendo então de dimensões mesoporosas, como anteriormente constatado pela isoterma.

A Fig. 4 exibe a morfologia da amostra Etil obtida

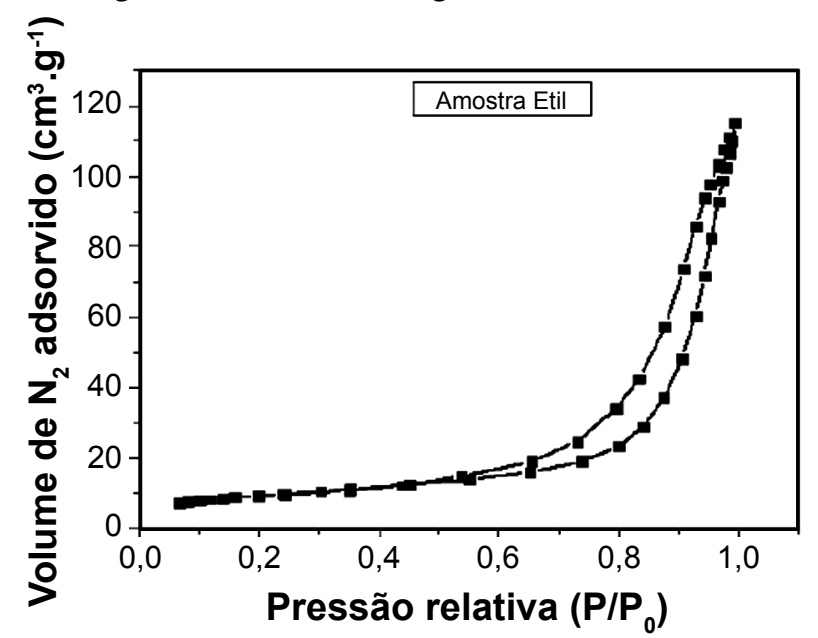

Figura 3: Isoterma de adsorção e dessorção da amostra Etil.

[Figure 3: Isotherm of adsorption and desorption of the Ethyl sample.] por microscopia eletrônica de varredura. Por meio das micrografias, verificou-se que as partículas estavam agregadas como consequência da sua reatividade, relacionada ao caráter nanométrico das partículas produzidas. As partículas agregadas possuíram uma morfologia aparentemente esférica, porém não bem definida. Percebeu-se que a queima a $1200{ }^{\circ} \mathrm{C}$ provocou uma pré-sinterização das partículas, evidenciando a alta reatividade do pó de mulita obtido pelo método Pechini. Os agregados apresentaram certa porosidade, com tamanhos de poros aparentemente da ordem de dezenas de nanômetros. A microestrutura obtida consistiu de uma grande quantidade de grãos equiaxiais, como também observado por outros autores que sintetizaram mulita por sol-gel $[26,52]$.

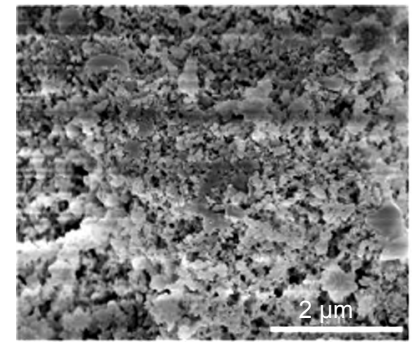

a)

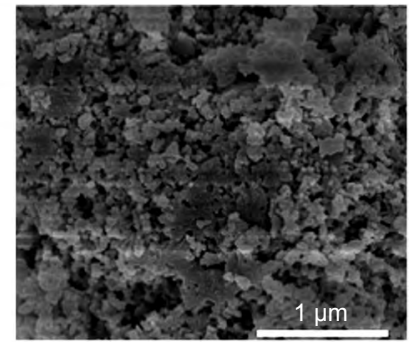

b)
Figura 4: Micrografias eletrônicas de varredura da amostra Etil. [Figure 4: Scanning electron micrographs of the Ethyl sample.]

As Figs. 5a e 5b exibem a micrografia eletrônica de transmissão e o padrão de difração de elétrons da amostra Etil, respectivamente. Observou-se que a amostra Etil foi constituída de numerosos nanocristais de formato esférico bem definido com tamanho estimado de cerca de $20 \mathrm{~nm}$, volumetricamente bem distribuídos, apresentando certa porosidade, e estavam parcialmente cobertos por uma fase amorfa. O padrão de difração de elétrons apresentou pontos de difração relacionados com a difração da região cristalina, mulita, além dos anéis difusos referentes à fase amorfa. Estes resultados foram em concordância com a difração de raios X e com o valor de cristalinidade, onde se obteve um padrão cristalino, porém com grande quantidade de material amorfo.

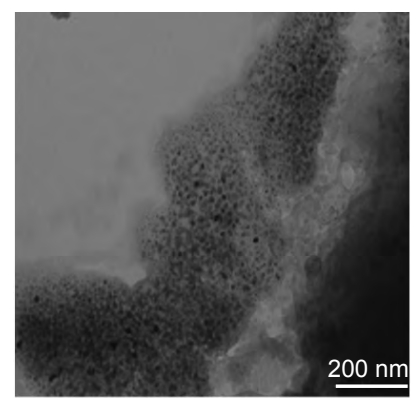

a)

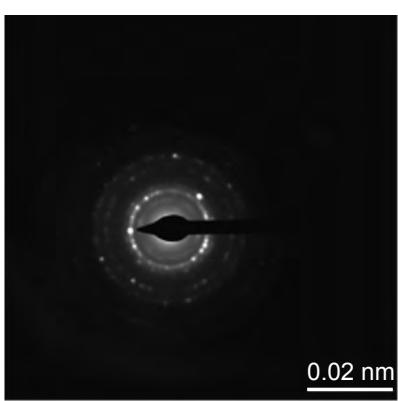

b)
Figura 5: Micrografia eletrônica de transmissão (a) e padrão de difração de elétrons (b) da amostra Etil.

[Figure 5: Transmission electron micrograph (a) and electron diffraction pattern $(b)$ of the Ethyl sample.] 


\section{CONCLUSÕES}

Diante dos resultados obtidos concluiu-se que o método Pechini é eficaz na obtenção da mulita, dependendo do solvente utilizado. O uso da água como solvente, seja esta usada em conjunto com o álcool etílico e/ou alterando-se seu $\mathrm{pH}$, dificultou a formação da mulita em decorrência da rápida hidrólise do TEOS. O álcool isopropílico usado como solvente na síntese retardou a formação da mulita, pela maior dificuldade de dissociação do ácido cítrico. A mulita foi obtida como fase majoritária e com maior grau de cristalinidade quando utilizado o álcool etílico como solvente, onde a amostra apresentou um valor de área superficial de $33,44 \mathrm{~m}^{2} / \mathrm{g}$, sendo constituída por partículas nanométricas que apresentaram alta reatividade, formando uma estrutura com certa porosidade.

\section{REFERÊNCIAS}

[1] I. Jaymes, A. Douy, D. Massiot, J.P. Coutures, J. Mater. Sci. 31 (1996) 4581.

[2] H. Schneider, J. Schreuer, B. Hildmann, J. Eur. Ceram. Soc. 28 (2008) 329.

[3] M. Asghari, T. Mohammadi, A. Aziznia, M.R. Danayi, S.H. Moosavi, R.F. Alamdari, F. Agand, Desalination 220 (2008) 65.

[4] A. Esharghawi, C. Penot, F. Nardou, J. Eur. Ceram. Soc. 29 (2009) 31.

[5] H. Zhang, Y. Hang, Y. Qin, J. Yang, W. Bo, J. Eur. Ceram. Soc. 34 (2014) 465.

[6] S. Sembiring, W. Simanjuntak, P. Manurung, D. Asmi, I.M. Low, Ceram. Int. 40 (2014) 7067.

[7] O. Khatim, T.H.N. Nguyen, M. Amamra, L. Museur, A. Khodan, A. Kanaev, Acta Mater. 71 (2014) 108.

[8] P.C. Yu, Y.W. Tsai, F.S. Yen, C.L. Huang, J. Am. Ceram. Soc. 97 (2014) 2431.

[9] S. Ilíc, S. Zec, M. Miljkovic, D. Poleti, M.P. Markovic, D.J. Janac'kovic, B. Matovic, J. Alloys Compd. 612 (2014) 259.

[10] L. Ren, Z. Fu, Y. Wang, F. Zhang, J. Zhang, W. Wang, H. Wang, J. Eur. Ceram. Soc. 35 (2015) 1915.

[11] S. Islam, N. Bidin, S. Riaz, S. Naseem, M.M. Sanagi, J. Taiwan Inst. Chem. Eng. 70 (2017) 366.

[12] A. Maho, S. Detriche, J. Delhalle, Z. Mekhalif, Mater. Sci. Eng. C 33 (2013) 2686.

[13] N. Bayal, P. Jeevanandam, Ceram. Int. 40 (2014) 15463. [14] L. Körösi, A. Scarpellini, P. Petrik, S. Papp, I. Dékány, Appl. Surf. Sci. 320 (2014) 725.

[15] R.R. Bhosale, Ceram. Int. 44 (2018) 8679.

[16] R.H. Jarman, J. Bafia, T. Gebreslasse, B.J. Ingram, J.D. Carter, Mater. Res. Bull. 48 (2013) 3916.

[17] A.N.S. Braga, A.C.G. Filho, L.S. Neiva, T.R. Silva, J.B.L. Oliveira, L. Gama, Mater. Sci. Forum 775 (2014) 678. [18] F.R. Cesconetoa, M. Borlafa, M.I. Nieto, A.P.N. Oliveira, R. Moreno, Ceram. Inter. 44 (2018) 301.

[19] M.P. Pechini, U.S. Patent 3330697 (1967).

[20] M. Kakihana, M. Yoshimura, Bull. Chem. Soc. Jpn. 72
(1999) 1427.

[21] A. Mashreghi, F. Davoudi. Mater. Sci. Semicond. Process. 30 (2015) 618.

[22] M.L Santos, A.O. Florentino, M.J. Saeki, A.H. Aparecida, M.V.L. Fook, A.C. Guastaldi, Eclet. Quim. 30 (2005) 29.

[23] M. Chrunik, A. Majchrowski, D. Zasada, A. Chlanda, M. Szala, M. Salerno, J. Alloys Compd. 725 (2017) 587.

[24] M. Ge, H. Yang, Z. Jiang, Y. Wang, F. Zhang, J. NonCryst. Solids 147 (1992) 565.

[25] D.J. Janackovic, A. Orlovic, D. Skala, S. Drmanic, L.J. Kostic-Gvozdenovic, V. Jokanovic, D. Uskokovic, Nanostruct. Mater. 12 (1999) 147.

[26] E.R. Sola, F.J. Torres, J. Alarcon, J. Eur. Ceram. Soc. 26 (2006) 2279.

[27] J. Leivo, M. Linden, J.M. Rosenholm, M. Ritola, C.V. Teixeira, E. Levanen, T.A. Mantyla, J. Eur. Ceram. Soc. 28 (2008) 1749.

[28] L.S. Cividanes, T.M.B. Campos, C.A. Bertran, G.P. Thim, J. Non-Cryst. Solids 356 (2009) 3013.

[29] K. Yoshida, H. Hyuga, N. Kondo, H. Kita, Mater. Sci. Eng. B 173 (2010) 66.

[30] V. Mandić, S. Kurajica. Mater. Sci. Semicond. Process. 38 (2015) 306.

[31] K. Yoshida, H. Hyuga, N. Kondo, H. Kita, Mater. Sci. Eng. B 173 (2010) 66.

[32] S. Kurajica, E. Tkalcec, V. Mandic, J. Schmauch, J. Eur. Ceram. Soc. 31 (2011) 377.

[33] D. Zeng, H. Zhang, J. Yang, B. Wang, X. Zhang, Ceram. Inter. 42 (2016) 11270.

[34] M.N. Rahaman, in $2^{\text {nd }}$ Ceram. Process. Sinter., Marcel Dekker, New York (2006).

[35] A.K. Chakraborty, J. Mater. Sci. 29 (1994) 6131.

[36] T.C. Oliveira, C.A. Ribeiro, D.D. Brunelli, L.A. Rodrigues, G.P. Thim, J. Non-Cryst. Solids 356 (2010) 2980. [37] G.P. Thim, C.A. Bertran, V.E. Barlette, M.I.F. Macedo, M.A.S. Oliveira, J. Eur. Ceram. Soc. 21 (2001) 759.

[38] T.M.B. Campos, L.S. Cividanes, D.D. Brunelli, K.K. Sakane, G.P. Thim, J. Eur. Ceram. Soc. 32 (2012) 835.

[39] K.H. Prasad, S. Subramanian, T.N. Sairam, G. Amarendra, E.S. Srinadhu, N. Satyanarayana, J. Alloys Compd. 718 (2017) 459.

[40] M.R. Loghman-Estarki, S. Torkian, R.A. Rastabi, A. Ghasemi, J. Magn. Magn. Mater. 442 (2017) 163.

[41] J.V. Reis, T.C.P. Pereira, T.H.A. Teles, A.B. França, J.D.A. Bellido, F.L. Naves, E.P. Baston, Mater. Lett. 227 (2018) 261.

[42] P. Fang, Z. Xi, W. Long, X. Li, J. Alloys Compd. 657 (2016) 273.

[43] A.K. Chakraborty, Thermochim. Acta 427 (2005) 109.

[44] K.S.W. Sing, Pure Appl. Chem. 54 (1982) 2201.

[45] K.S.W. Sing, R.T. Williams, Adsorpt. Sci. Technol. 22 (2004) 773.

[46] P. Klobes, K. Meyer, R.G. Munro, "Porosity and specific surface area measurements for solid materials", NIST, USA (2006).

[47] K.S.W. Sing, D.H. Everett, R.A.W. Haul, L. Moscou, 
R.A. Pierotti, J. Rouquerol, T. Siemieniewska, Pure Appl. Chem. 57 (1985) 603.

[48] J.L. Figueiredo, F.R. Ribeiro, Catálise heterogênea, Ed. Fund. Calouste Gulbenkian, Lisboa (1987).

[49] A.E. Ahmed, F. Adam, Micropor. Mesopor. Mater. 103 (2007) 284.
[50] F. Adam, J.N. Appaturi, R. Thankappan, M.A.M. Nawi, Appl. Surf. Sci. 257 (2010) 811.

[51] X. Guo, W. Li, K. Nakanishi, K. Kanamori, Y. Zhu, H. Yang, J. Eur. Ceram. Soc. 33 (2013) 1967.

[52] G. Zhang, Z. Fu, Y. Wang, H. Wang, W. Wang, J. Zhang, S.W. Lee, K. Niihara, J. Eur. Ceram. Soc. 30 (2010) 2435.

(Rec. 24/09/2018, Rev. 20/12/2018, Ac. 19/02/2019) 